\title{
Single- and multi-wall carbon nanotube field-effect transistors
}

\author{
R. Martel, T. Schmidt, H. R. Shea, T. Hertel, and Ph. Avouris ${ }^{\text {a) }}$ \\ IBM Research Division, T. J. Watson Research Center, Yorktown Heights, New York 10598
}

(Received 1 July 1998; accepted for publication 24 August 1998)

\begin{abstract}
We fabricated field-effect transistors based on individual single- and multi-wall carbon nanotubes and analyzed their performance. Transport through the nanotubes is dominated by holes and, at room temperature, it appears to be diffusive rather than ballistic. By varying the gate voltage, we successfully modulated the conductance of a single-wall device by more than 5 orders of magnitude. Multi-wall nanotubes show typically no gate effect, but structural deformations-in our case a collapsed tube-can make them operate as field-effect transistors. (C) 1998 American Institute of Physics. [S0003-6951(98)00143-0]
\end{abstract}

Carbon nanotubes (NTs) are a new form of carbon with unique electrical and mechanical properties. ${ }^{1}$ They can be considered as the result of folding graphite layers into carbon cylinders and may be composed of a single shell-single wall nanotubes (SWNTs), or of several shells-multi-wall nanotubes (MWNTs). Depending on the folding angle and the diameter, nanotubes can be metallic or semiconducting. Simple theory also shows that the band gap of semiconducting NTs decreases with increasing diameter. These predictions have been verified in recent scanning tunneling spectroscopy experiments. ${ }^{2,3}$

Their interesting electronic structure makes carbon nanotubes ideal candidates for novel molecular devices. Metallic NTs, for example, were utilized as Coulomb islands in single-electron transistors ${ }^{4,5}$ and, very recently, Tans and coworkers built a molecular field-effect transistor (FET) with a semiconducting nanotube. ${ }^{6}$

In this letter, we report on the fabrication and performance of a SWNT-based FET and explore whether MWNTs can be utilized as the active element of carbon-based FETs. Despite their large diameter, we find that structurally deformed MWNTs may well be employed in NT-FETs. Based on the output and transfer characteristics of our NT devices, we evaluate their carrier density and discuss the transport mechanism.

The SWNTs used in our study were produced by laser ablation of graphite doped with cobalt and nickel catalysts. ${ }^{7}$ For cleaning, the SWNTs were ultrasonically treated in an $\mathrm{H}_{2} \mathrm{SO}_{4} / \mathrm{H}_{2} \mathrm{O}_{2}$ solution. MWNTs were produced by an arcdischarge evaporation technique ${ }^{8}$ and used without further treatment. The NTs were dispersed by sonication in dichloroethane and then spread on a substrate with predefined electrodes. A schematic cross section of a NT device is shown in Fig. 1. They consist of either an individual SWNT or MWNT bridging two electrodes deposited on a $140 \mathrm{~nm}$ thick gate oxide film on a doped $\mathrm{Si}$ wafer, which is used as a back gate. The $30 \mathrm{~nm}$ thick Au electrodes were defined using electron beam lithography. For imaging, we used an atomic force microscope operating in the noncontact mode. The sourcedrain current $I$ through the NTs was measured at room tem-

${ }^{a)}$ Electronic mail: avouris@us.ibm.com perature as a function of the bias voltage $V_{\mathrm{SD}}$ and the gate voltage $V_{G}$.

Figure 2(a) shows the output characteristics $I-V_{\mathrm{SD}}$ of a device consisting of a single SWNT with a diameter of 1.6 $\mathrm{nm}$ for several values of the gate voltage. At $V_{G}=0 \mathrm{~V}$, the $I-V_{\mathrm{SD}}$ curve is linear with a resistance of $R=2.9 \mathrm{M} \Omega$. For $V_{G}<0 \mathrm{~V}$, the $I-V_{\mathrm{SD}}$ curves remain linear, whereas they become increasingly nonlinear for $V_{G} \gg 0 \mathrm{~V}$ up to a point where the current becomes unmeasurably small, indicating a controllable transition between a quasimetallic and an insulating state of the NT. Figure 2(b) shows transfer characteristics $I-V_{G}$ of our NT device for different source-drain voltages. The behavior is similar to that of a $p$-channel metaloxide-semiconductor FET. ${ }^{9}$ The source-drain current decreases strongly with increasing gate voltage, which not only demonstrates that the NT device operates as a fieldeffect transistor but also that transport through the semiconducting SWNT is dominated by positive carriers (holes). The conductance modulation of our SWNT-FET exceeds 5 orders of magnitude [see inset of Fig. 2(b)]. For $V_{G}<0 \mathrm{~V}$, the $I-V_{G}$ curves saturate indicating that the contact resistance $R_{C}$ at the metal electrodes starts to dominate the total resistance $R=R_{\mathrm{NT}}+2 R_{C}$ of the device. Here, $R_{\mathrm{NT}}$ denotes the gate-dependent resistance of the NT. The saturation value of the current corresponds to $R_{C} \approx 1.1 \mathrm{M} \Omega$. Similar contact resistances were previously found for metallic SWNTs. ${ }^{4}$

The origin of the holes is an important question to address. One possibility is that the carrier concentration is inherent to the NT. Another possibility is that the majority of carriers are injected at the gold-nanotube contacts. The higher work function of gold leads to the generation of holes in the NT by electron transfer from the NT to the gold

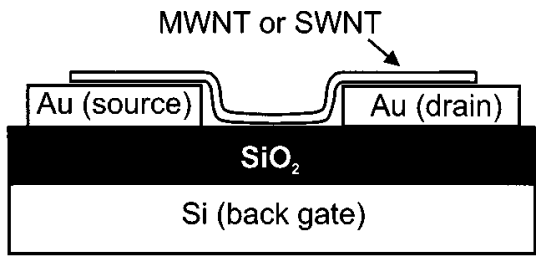

FIG. 1. Schematic cross section of the FET devices. A single NT of either MW or SW type bridges the gap between two gold electrodes. The silicon substrate is used as back gate. 

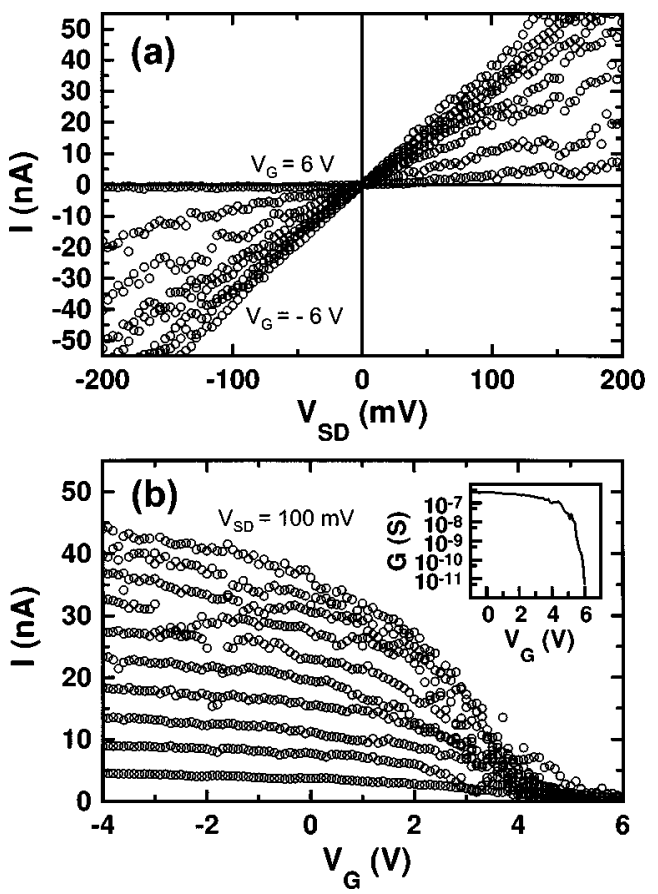

FIG. 2. Output and transfer characteristics of a SWNT-FET: (a) $I-V_{\mathrm{SD}}$ curves measured for $V_{G}=-6,0,1,2,3,4,5$, and $6 \mathrm{~V}$. (b) $I-V_{G}$ curves for $V_{\mathrm{SD}}=10-100 \mathrm{mV}$ in steps of $10 \mathrm{mV}$. The inset shows that the gate modulates the conductance by 5 orders of magnitude $\left(V_{\mathrm{SD}}=10 \mathrm{mV}\right)$.

electrodes. ${ }^{2}$ Assuming that the band-bending length in their SWNT is neither very short nor very long, Tans et al. have argued that the FET operation can be explained based on this charge transfer. ${ }^{6}$ At $V_{G}=0 \mathrm{~V}$, the device is "on" and the Fermi energy is close to the valence-band edge throughout the NT. If indeed the band-bending length is comparable to the length of the SWNT, a positive gate voltage would generate an energy barrier of an appreciable fraction of $e V_{G}$ in the center of the tube (since the gate/NT distance is shorter than the source/drain separation). The threshold voltage $V_{G, T}$ required to suppress hole conduction by depleting the tube center would be determined by the thermal energy available for overcoming this barrier. Thus, $V_{G, T}$ should be much lower than the $6 \mathrm{~V}$ observed in Fig. 2(b). Therefore, it is important to explore the other possibility, namely, that the carriers are an inherent property of the tube.

In this case, we expect a fairly homogeneous hole distribution along the NT independent of the gate voltage. An estimate of the hole density can then be obtained by writing the total charge on the NT as $Q=C V_{G, T}$, where $C$ is the NT capacitance and $V_{G, T}$ the threshold voltage necessary to completely deplete the tube. The NT capacitance per unit length with respect to the back gate is $C / L$ $\approx 2 \pi \epsilon \epsilon_{0} / \ln (2 h / r)$, with $r$ and $L$ being the NT radius and length, and $h$ and $\epsilon$ the thickness and the average dielectric constant of the device. ${ }^{10}$ Using $L=300 \mathrm{~nm}, r=0.8 \mathrm{~nm}, h$ $=140 \mathrm{~nm}$, and $\epsilon \approx 2.5$, we evaluate a one-dimensional hole density of $p=Q / e L \approx 9 \times 10^{6} \mathrm{~cm}^{-1}$ from $V_{G, T}=6 \mathrm{~V}$. This value corresponds to about 1 hole per 250 carbon atoms in the NT. For comparison, in graphite there is only 1 hole per $10^{4}$ atoms. ${ }^{11}$ The large hole density suggests that the NT is degenerate and/or that it is doped with acceptors, for example, as a result of its processing. ${ }^{12}$

Assuming that transport in our NT is diffusive at room Downloaded 23 Mar 2010 to 128.178 .201 .242 . Redistribution subject

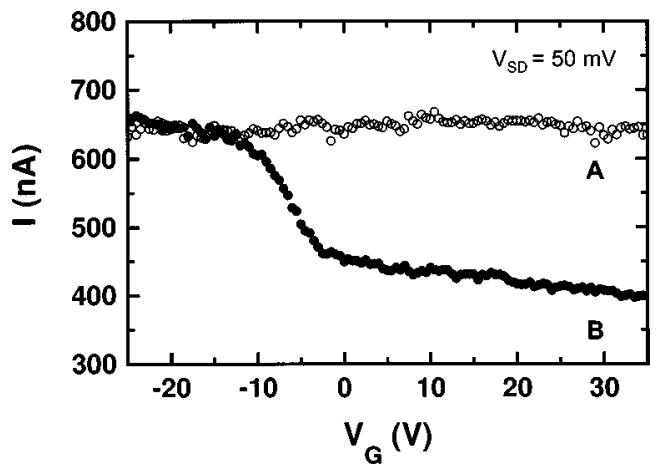

FIG. 3. $I-V_{G}$ curve of a typical MWNT device (curve A) in comparison with that of a collapsed MWNT of similar cross section (curve B).

temperature (as in SWNT bundles ${ }^{13}$ ), we can estimate the mobility of the holes from the transconductance of the FET. In the linear regime, it is given by $d I / d V_{G}$ $=\mu_{h}\left(C / L^{2}\right) V_{\mathrm{SD}}$. Subtracting the contact resistance, we obtain a NT transconductance of $d I / d V_{G}=1.7 \times 10^{-9} \mathrm{~A} / \mathrm{V}$ at $V_{\mathrm{SD}}=10 \mathrm{mV}$, corresponding to a hole mobility of $\mu_{h}$ $\approx 20 \mathrm{~cm}^{2} / \mathrm{V}$ s. This value is close to the mobility in heavily $p$-doped silicon of comparable hole density, ${ }^{9}$ but considerably smaller than the $10^{4} \mathrm{~cm}^{2} / \mathrm{V} \mathrm{s}$ observed in graphite. ${ }^{11}$ The low value of the NT mobility is consistent with our initial assumption of diffusive transport and suggests that the SWNT contains a large number of scatterers, possibly related to defects in the NT or disorder at the NT/gate-oxide interface due to roughness. SWNTs are known to conform to the topography of the surface so as to increase their adhesion energy. Such deformations can lead to local electronic structure changes, ${ }^{14}$ which may act as scattering centers. The low mobility is surprising in view of the coherence length of more than $1 \mu \mathrm{m}$ reported on the basis of energy quantization along a metallic SWNT at low temperature. ${ }^{4}$ However, we note that there have been no transport experiments on individual SWNTs that provide evidence for ballistic transport at room temperature (e.g., by observing conductance quantization). ${ }^{15}$

Having demonstrated FET operation for a SWNT, we move on to explore whether transport through MWNTs can be controlled by a gate electrode. The band gap of NTs has been predicted to decrease with increasing tube diameter. ${ }^{1}$ Therefore, MWNTs with diameters of $10 \mathrm{~nm}$ or more are expected to show metallic rather than semiconducting behavior at room temperature. We fabricated a number of MWNT devices with resistances of $R \sim 100 \mathrm{k} \Omega$. Most of these devices showed no gate action, and a typical $I-V_{G}$ curve is plotted in Fig. 3 (curve A).

Structural deformations of NTs change their electronic properties. Curve B in Fig. 3 shows that this can lead to a significant gate effect in MWNTs. As is the case for the SWNT-FET, the source-drain current of this MWNT-FET decreases with increasing gate voltage, i.e., the dominant conduction process is hole transport. In contrast to the SWNT device, this MWNT-FET could not be completely depleted. The $I-V_{\mathrm{SD}}$ curve remained linear independent of the gate voltage (not shown). Between $V_{G}=-35$ and $25 \mathrm{~V}$, the resistance increased only from $R=76$ to $120 \mathrm{k} \Omega$, corresponding to a conductance modulation by about a factor 2 . to AIP license or copyright; see http://apl.aip.org/apl/copyright.jsp 

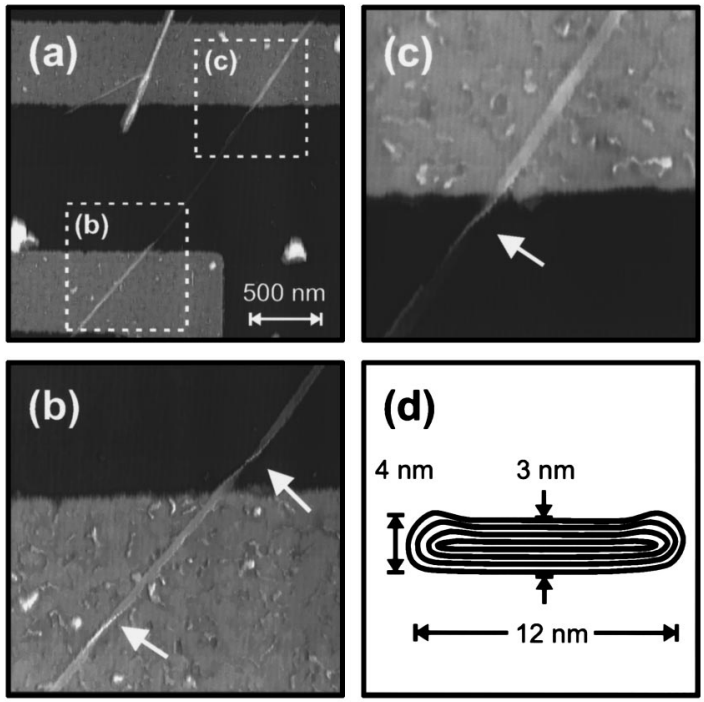

FIG. 4. (a) Noncontact AFM image of the MWNT-FET. (b) and (c) Close up views showing three twists in the collpased NT (see arrows). (d) Schematic cross section of the collapsed MWNT.

The gate effect reaches a sharp maximum between $V_{G}$ $=-15$ and $0 \mathrm{~V} \cdot{ }^{16}$

To explain this peculiar behavior, we consider the AFM image, Fig. 4(a) of the MWNT-FET. The device consists of a collapsed MWNT, which bridges the gap between two Au electrodes separated by about $1 \mu \mathrm{m}$. This nanostripe is $3 \mathrm{~nm}$ high-from which we conclude that it has four or five shells-and it exhibits a number of twists [Figs. 4(b) and (c)] — which allow us to determine its width to be $12 \mathrm{~nm}$.

Based on the structural information summarized in Fig. 4(d), we propose the following explanation for the behavior of the MWNT-FET. Since the intershell interaction in MWNTs is weak, it is reasonable to assume that transport is confined to the outermost shell of the nanostripe. ${ }^{15}$ The conductance modulation of about 2 indicates that the bottom "plate" of the outermost shell is depleted by the gate, whereas the top layer is less affected due to screening by the inner shells (and the bottom layer as long as it is conducting). Our model implies that the bottom "plate" is decoupled from the top layer, which may be the consequence of lateral quantization effects perpendicular to the tube axis. Using $R=R_{\mathrm{NT}}+2 R_{C}$ for the "on" state $\left(V_{G}=-15 \mathrm{~V}\right)$ and $R=2 R_{\mathrm{NT}}+2 R_{C}$ for the "off" state of the MWNT-FET $\left(V_{G}=0 \mathrm{~V}\right)$, we estimate a resistance of $R_{\mathrm{NT}}=32 \mathrm{k} \Omega$ for the outer shell of the NT and deduce a contact resistance of $R_{C}$ $=23 \mathrm{k} \Omega$.

Finally, we proceed analogously to the SWNT-FET analysis to evaluate the hole density and mobility of the collapsed MWNT. Numerical calculations show that the capacitance per unit length is reasonably well described by $C / L$ $=2 \pi \epsilon \epsilon_{0} / \ln (2 h / r)$ despite the slab-shaped geometry of the collapsed tube. Using $L=1.1 \mu \mathrm{m}, r=5 \mathrm{~nm}$, and a threshold voltage of $V_{G, T} \approx 8 \mathrm{~V}$ to deplete the bottom layer, we obtain $p \approx 1.7 \times 10^{7} \mathrm{~cm}^{-1}$ for its hole density. From the transconductance of $d I / d V_{G}=3.5 \times 10^{-8} \mathrm{~V} / \mathrm{A}$ at $V_{\mathrm{SD}}=50 \mathrm{mV}$, we estimate a mobility of $\mu_{h} \approx 220 \mathrm{~cm}^{2} / \mathrm{V}$ s. The hole density is similar to the SWNT but the mobility is higher, which suggests a reduced number of scatterers. This may arise from the fact that the MWNTs were not ultrasonically treated in acids. Furthermore, they do not deform as much as SWNTs in order to conform to roughness at the NT/gate-oxide interface.

In conclusion, we fabricated molecular field-effect transistors with single- and multi-wall carbon nanotubes. Transport in the NTs is dominated by holes and, at room temperature, it appears to be diffusive. Using the gate electrode, the conductance of a SWNT-FET could be modulated by more than 5 orders of magnitude. An analysis of the transfer characteristics of the FETs suggests that the NTs have a higher carrier density than graphite and a hole mobility comparable to heavily $p$-doped silicon. Large-diameter MWNTs show typically no gate effect, but structural deformations can modify their electronic structure sufficiently to allow FET behavior.

The authors thank A. G. Rinzler, R. E. Smalley, and H. Dai for providing the NTs, and they gratefully acknowledge S. Koester and M. J. Rooks for assistance with the electronbeam lithography, F. Stern for capacitance calculations, and P. Solomon for helpful discussions. T.S. and T.H. thank the Alexander von Humboldt Foundation for support.

${ }^{1}$ For a review, see M. S. Dresselhaus, G. Dresselhaus, and P. C. Eklund, Science of Fullerenes and Carbon Nanotubes (Academic, San Diego, CA, 1996).

${ }^{2}$ J. W. G. Wildöer, L. C. Venema, A. G. Rinzler, R. E. Smalley, and C. Dekker, Nature (London) 391, 59 (1998).

${ }^{3}$ T. W. Odom, J.-L. Huang, P. Kim, and C. M. Lieber, Nature (London) 391, 62 (1998).

${ }^{4}$ S. J. Tans, M. H. Devoret, H. Dai, A. Thess, R. E. Smalley, L. J. Geerligs, and C. Dekker, Nature (London) 386, 474 (1997).

${ }^{5}$ M. Bockrath, D. H. Cobden, P. L. McEuen, N. G. Chopra, A. Zettl, A. Thess, and R. E. Smalley, Science 275, 1922 (1997).

${ }^{6}$ S. J. Tans, A. R. M. Verschueren, and C. Dekker, Nature (London) 393, 49 (1998).

${ }^{7}$ T. Guo, P. Nikolaev, A. Thess, D. T. Colbert, and R. E. Smalley, Chem. Phys. Lett. 243, 49 (1995).

${ }^{8}$ D. T. Colbert, J. Zhang, S. M. McClure, P. Nikolaev, J. H. Hafner, D. W. Owens, P. G. Kotula, C. B. Carter, J. H. Weaver, A. G. Rinzler, and R. E. Smalley, Science 266, 1218 (1994).

${ }^{9}$ S. M. Sze, Physics of Semiconductor Devices (Wiley, New York, 1981).

${ }^{10}$ This expression was inferred from P. M. Morse and H. Feshbach, Methods of Theoretical Physics (McGraw-Hill, New York, 1953), p. 11. The NT is considered as a metallic cylinder, which is a good approximation as long as the density of states at the Fermi level is high. Similar expressions were used to estimate Coulomb-blockade energies of NTs.

${ }^{11}$ N. B. Brandt, S. M. Chudinov, and Ya. G. Ponomarev, Semimetals, 1. Graphite and its Compounds (North-Holland, Amsterdam, 1988).

${ }^{12} \mathrm{H}$. He, J. Klinowski, M. Forster, and A. Lerf, Chem. Phys. Lett. 287, 53 (1998).

${ }^{13}$ J. E. Fischer, H. Dai, A. Thess, R. Lee, N. M. Hanjani, D. L. Dehaas, and R. E. Smalley, Phys. Rev. B 55, R4921 (1997).

${ }^{14}$ A. Rochefort, D. Salahub, and Ph. Avouris, Chem. Phys. Lett. (to be published).

${ }^{15}$ Only in freely suspended MWNTs without NT/substrate interaction has evidence for ballistic room-temperature transport been found [S. Frank, P. Poncharal, Z. L. Wang, and W. A. de Heer, Science 280, 1744 (1998)].

${ }^{16}$ The position of the sharp drop in the $I-V_{G}$ curve was found to depend on the sweep direction, but the voltage range over which the drop occurs showed no hysteresis. 